\title{
Total Knee Arthroplasty in Spondyloepiphyseal Dysplasia with Irreducible Congenital Dislocation of the Patella: Case Report and Literature Review
}

This article was published in the following Dove Press journal:

Therapeutics and Clinical Risk Management

\section{Pavel Sponer (iD \\ Martin Korbel \\ Tomas Kucera (DD}

Department of Orthopedic Surgery, Charles University, Faculty of Medicine in Hradec Kralove, Hradec Kralove, Czech Republic
Correspondence: Pavel Sponer

Department of Orthopedic Surgery,

Charles University, Faculty of Medicine in

Hradec Kralove, Hradec Kralove, 500 03,

Czech Republic

Tel +420 495833566

Fax +420 495832007

Email sponer.p@seznam.cz
Background: Spondyloepiphyseal dysplasia is the clinical term applied to a group of rare genetic disorders with primary involvement of the vertebrae and epiphyses, predisposing the afflicted individuals toward the premature development of osteoarthritis. There are few reports concerning joint replacement therapy in these patients, particularly describing the role of total hip arthroplasty. In this report, we describe the anatomical and technical aspects of spondyloepiphyseal dysplasia that must be considered during surgical planning and performance of total knee arthroplasty.

Case Presentation: A 49-year old woman with a history of spondyloepiphyseal dysplasia suffered from severe osteoarthritis of the knee and irreducible congenital dislocation of the patella. After careful preoperative evaluations and planning, the knee joint deformity was solved by knee joint replacement with realignment of the extensor mechanism using quadricepsplasty. After 2 years of surgery, the patient showed no pain and was able to walk with the help of elbow crutches. The Hospital for Special Surgery knee score increased from preoperative 51 points to 85 points during the final follow-up. The postoperative range of motion increased to final flexion of $0-115^{\circ}$.

Conclusion: The advances made so far in the medical care for patients with skeletal dysplasia have improved their overall survival during adulthood. The case report described herein demonstrates the numerous challenges and technical aspects of a successful total knee arthroplasty in cases of spondyloepiphyseal dysplasia, highlighting the need to consider skeletal and soft tissue abnormalities of skeletal dysplasia during the planning and performance of joint replacement surgery.

Keywords: spondyloepiphyseal dysplasia, patella dislocation, total knee arthroplasty

\section{Introduction}

Skeletal dysplasias are a group of more than 450 heterogeneous disorders characterized by the abnormal growth and remodeling of bone and cartilage and whose diagnosis is typically made through radiographic, clinical, and molecular criteria. ${ }^{1}$ Among the types of skeletal dysplasias, spondyloepiphyseal dysplasia includes those disorders with the primary involvement of both vertebrae and epiphyses, which is caused by the abnormal synthesis of Type II collagen. Thus, these types of disorders result in disproportionate short-trunk dwarfism and abnormal epiphyseal development. $^{2}$ Abnormal bone and joint development, in conjunction with altered biomechanics, predisposes spondyloepiphyseal dysplasia patients toward the premature development of osteoarthritis, especially in hip and knee joints. ${ }^{3,4}$ In the 
case of children, joint preservation techniques, including osteotomies to achieve an upper femur and sagittal spinopelvic radiographic alignment, are recommended to improve hip pain and range of motion. ${ }^{5}$ However, alterations in development, anatomy, and biomechanics may require joint replacement surgery during the young adult age. $^{6}$

Joint replacement in skeletal dysplasia population is a technically challenging procedure due to the abnormal anatomy of the patient, eg, bone deformities, small bone caliber, axis deviation, ligament laxity, and soft tissue contractures. In addition, the young age of the patients imposes the selection of the implant based on its longterm survival. ${ }^{7}$ The available literature on joint replacement in these cases is limited by the heterogeneity of series evaluating multiple forms of skeletal dysplasia or different locations of joint replacement surgeries in selected skeletal dysplasia. ${ }^{4,7,8}$ There are only few reports demonstrating significant pain reduction, improved function, and independent movement following total hip arthroplasty in patients with spondyloepiphyseal dysplasia., $^{3,9}$

Each type of skeletal dysplasia has its own unique pathological characteristics. However, none of the available studies evaluating total knee arthroplasty in multiple forms of skeletal dysplasia describe the surgical technique in patients with spondyloepiphyseal dysplasia., ${ }^{4,7}$ Skeletal and soft tissue knee abnormalities that are typical for spondyloepiphyseal dysplasia should be taken into account during the surgical planning and performance of joint replacement surgery. In this case report, we describe a patient with spondyloepiphyseal dysplasia who suffered from severe osteoarthritis of the knee and irreducible congenital dislocation of the patella solved by knee joint replacement with realignment of the extensor mechanism using quadricepsplasty. In addition, we review the existing literature and present technical observations on the management of this rare scenario.

\section{Case History}

A 49-year old woman with a history of spondyloepiphyseal dysplasia presented right knee deformity that became symptomatic after a right hip joint replacement performed one year earlier. She complained of right knee pain and reduced walking distance secondary to knee osteoarthritis. At the age of eight years, soft tissue release was performed for flexion contracture of the right knee. Two years later, she was diagnosed with unilateral congenital dislocation of right patella and was treated nonoperatively. Physical examination showed bilateral genu valgum deformity. The right knee had an extension lag of $15^{\circ}$ and range of flexion to $150^{\circ}$. The right medial collateral ligament had moderate laxity. Her right patella was laterally dislocated. At $90^{\circ}$ of flexion, the right tibia, including tibial tuberosity and anterior aspect of ankle joint, was rotated $30^{\circ}$ externally. This rotational deformity in flexion was partially correctable in a passive manner. The patient was not able to actively extend her right knee against gravity, peripheral pulses were intact. A long-standing anteroposterior radiograph confirmed $16^{\circ}$ of genu valgum deformity with the mechanical axis passing through her lateral tibial plateau (Figures 1 and 2). A CT scan evaluation revealed a narrow medullary cavity of the distal femur as well as patellar dislocation and severe osteoarthritis of the knee with complete loss of the lateral compartment joint space (Figure 3). Imaging tests disclosed gross osteopenia. The patient was $149-\mathrm{cm}$ tall with a body mass index value of $23 \mathrm{~kg} / \mathrm{m}^{2}$. The medical comorbidities included hypertension, osteoporosis and severe thoracolumbar scoliosis. The patient did not smoke. The patient elected to undergo right total knee arthroplasty after discussing the benefits and risks of the surgery.

On the day of surgery, a midline longitudinal skin incision was made without tourniquet inflation. The vastus medialis muscle was found in an anterior position covering the femoral condyles in consequence of lateral dislocation of the extensor mechanism. The inferior border of the vastus medialis was dissected off the underlying joint capsule and a dissection was continued underneath the vastus medialis proximally. The patella was palpable on the lateral aspect of the lateral femoral condyle (Figure 4). The tibial tubercle and medial border of the patella were marked with stitches. The medial parapatellar arthrotomy was performed with the vertical limb of the incision taken along the medial edge of the patellar tendon to the tibial tubercle and the horizontal limb of the incision made along the inferior margin of the vastus medialis. Retracting the quadriceps laterally, the patella and the vastus lateralis were identified and lateral patellar retinaculum release was performed from inside the joint staying lateral to the vastus lateralis. Both cut ends of the superior lateral genicular artery were cauterized.

Total knee arthroplasty was done following a measured resection technique by conventional intramedullary femoral guide. The distal part of the femur was osteotomized at $6^{\circ}$ of valgus. The rotation of the femur was based 


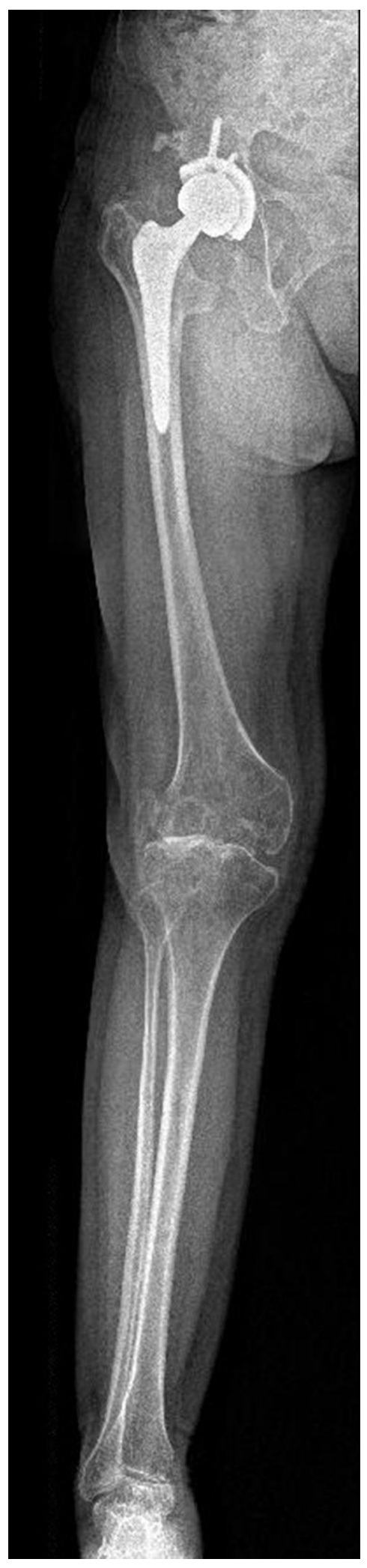

Figure I Preoperative long-standing anteroposterior radiograph of the lower extremity.

on the transepicondylar axis. After the proximal tibial cut, the intramedullary tibial cutting guide was removed to

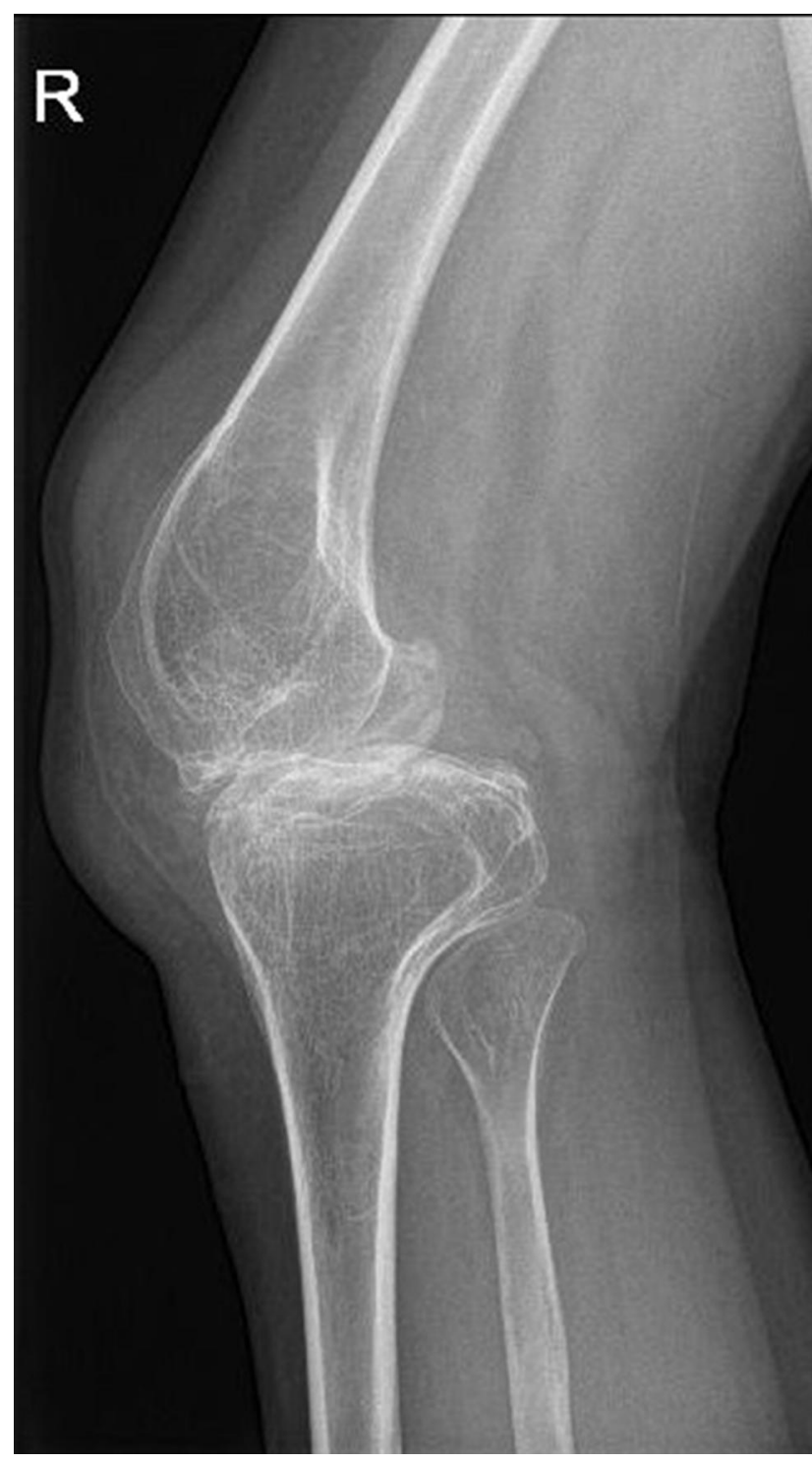

Figure 2 Preoperative lateral radiograph of the right knee.

assess the extension gap soft tissue balancing. With regard to the identified medial instability of the knee joint, a constrained condylar knee implant (NexGen Legacy Constrained Condylar Knee, Zimmer, Warsaw, Indiana, USA) was chosen for this case. Given the presence of metaphyseal osteopenia, an uncemented 100-mm stem extension was used to augment fixation in the tibia. The shaft was reamed gently without complications. The rotational alignment of the tibial component was determined with reference to the middle of the tibial tubercle. Femoral chamfer and box cuts were made after sizing the distal femur. Considering the osteopenic change in the femoral condyles, the small diameter of the medullary cavity, and a sufficient interprosthetic distance, a cemented 30-mm 


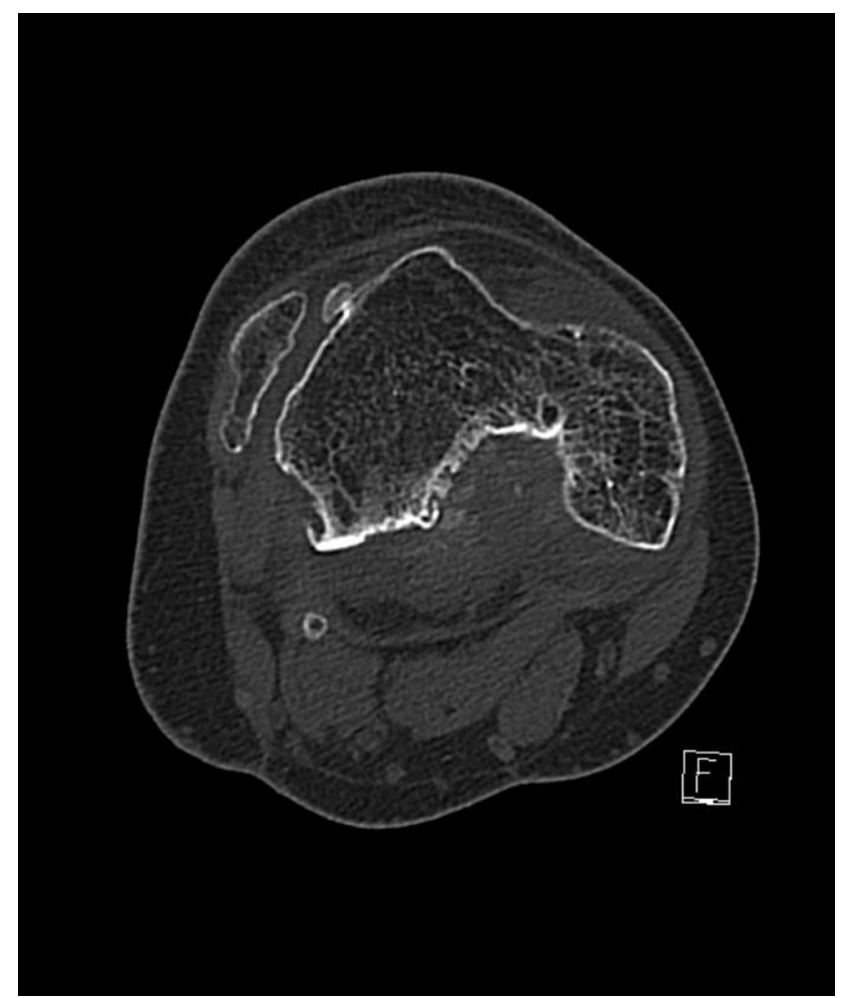

Figure 3 Patellar dislocation on preoperative CT scan.

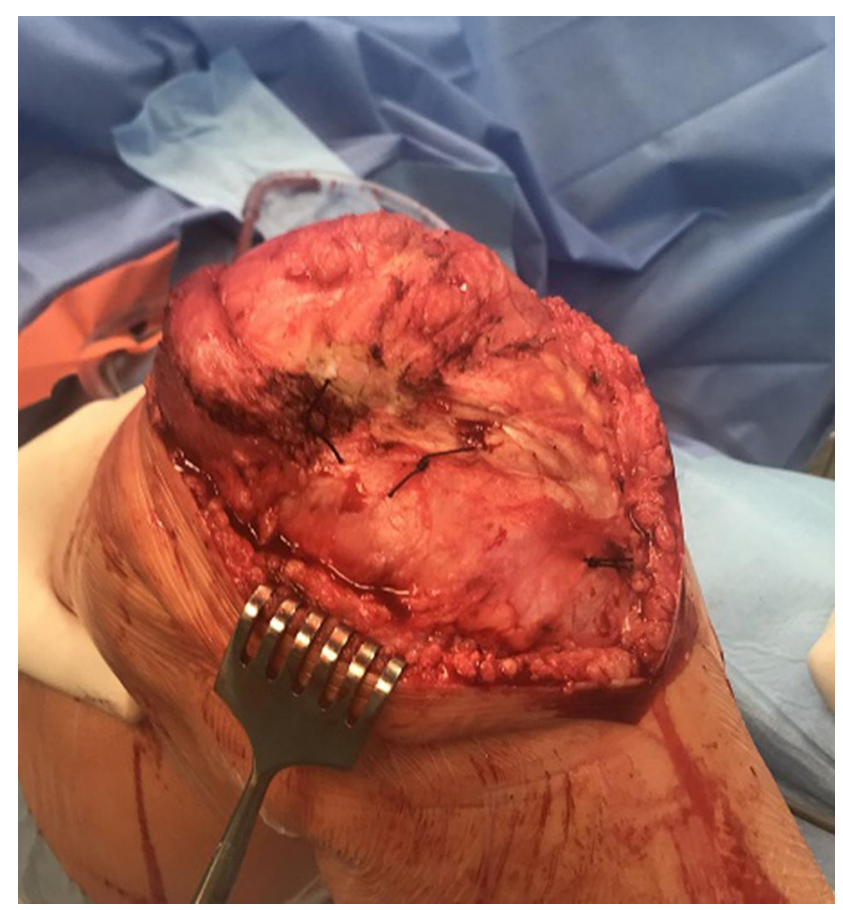

Figure 4 Intraoperative photography showing the patella located on the lateral aspect of the lateral femoral condyle (medial border of patella was marked with two stitches).

stem extension was selected to increase the fixation of the femoral component. Both trial components with stems fitted successfully and in correct rotational alignment; however, good patella tracking could not be achieved due to the tension of the vastus lateralis muscle.

After proximal extension of the skin incision, the vastus lateralis was released from the lateral intermuscular septum and anterior femur by submuscular dissection. Afterwards, the quadriceps tendon and patella were centralized with passive knee flexion. Patella resurfacing was not performed because of its small size, only a prominent lateral osteophyte was removed. The definitive components were cemented (Figure 5). At this point, the knee was flexed $90^{\circ}$, and the fibrous capsular flap originating in the space between the adductor tubercle and the medial femoral epicondyle was temporarily fixed to the superolateral aspect of the patella. The condition of the knee was assessed through a $0-100^{\circ}$ range of motion confirming central patellar tracking. The distal part of the fibrous capsular flap was brought over the patellar tendon and the redundant pouch of the capsule was resected. The distal medial repair tied the capsule and the patellar ligament in a secure manner. The proximal part of the fibrous capsular flap was then pulled over the anterior

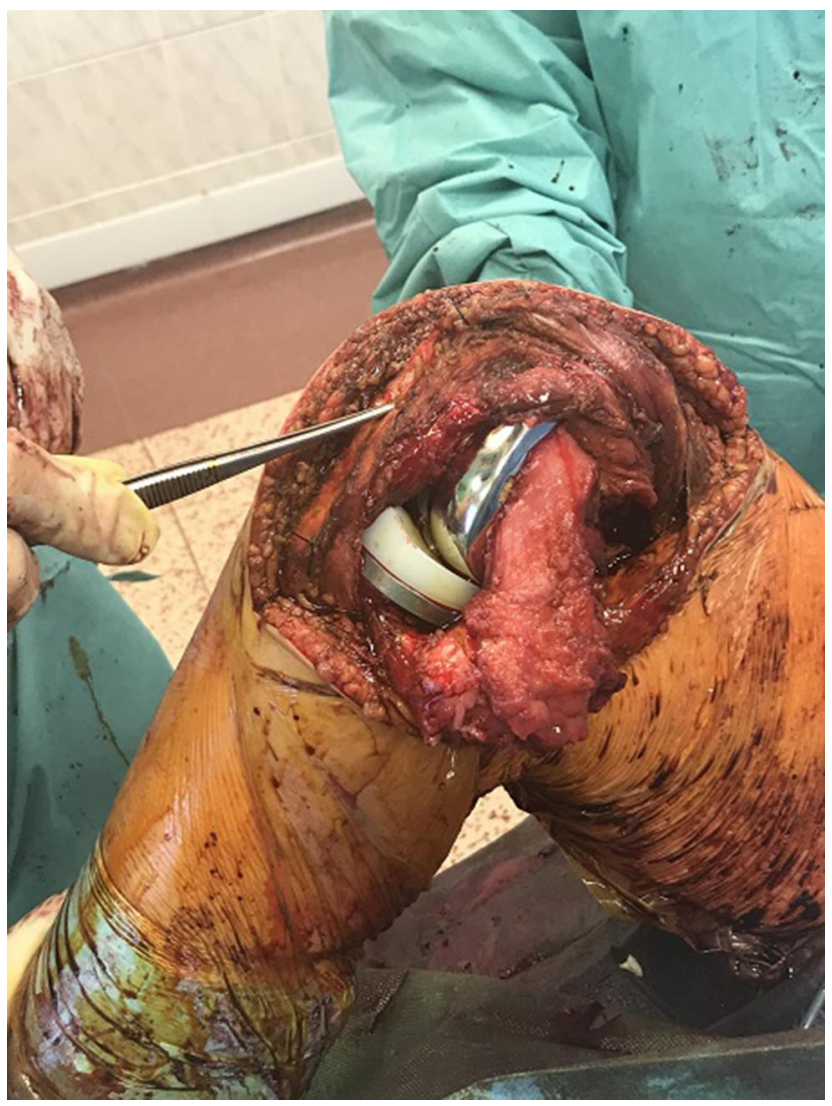

Figure 5 Intraoperative photography showing the patella centrally placed in the groove of the femoral component. 
aspect of the patella and sutured to the extensor apparatus. The medial capsular suture was completed proximally and the wound was closed with suction drain. The intraoperative range of motion was of $0-100^{\circ}$. Schematic diagrams present a step-by-step description of the surgical technique (Figure $6 \mathrm{~A}$ and $\mathrm{B})$.

Antibiotic prophylaxis lasted for 24 hours and lowmolecular-weight heparin was administered to prevent thromboembolic disease 5 weeks after surgery. Physiotherapy began at post-operative day 1. Partial weight-bearing was allowed immediately after surgery, proceeding to a full weight-bearing walk after 6 weeks.
At the 6-week follow-up visit, the patient reported pain complete resolution and was able to actively extend her right knee against gravity. She had a painless range of flexion from 5 to $90^{\circ}$ and ongoing physiotherapy was prolonged. After 2 years, the patient had no pain and ambulated with the help of elbow crutches. The preoperative Hospital for Special Surgery knee score increased from preoperative 51 points to 85 points at final followup. The postoperative range of motion increased to final flexion of $0-115^{\circ}$. Anteroposterior and lateral radiographs were obtained, the patella was clearly seen on the lateral view (Figure 7A and B). The patient has provided written
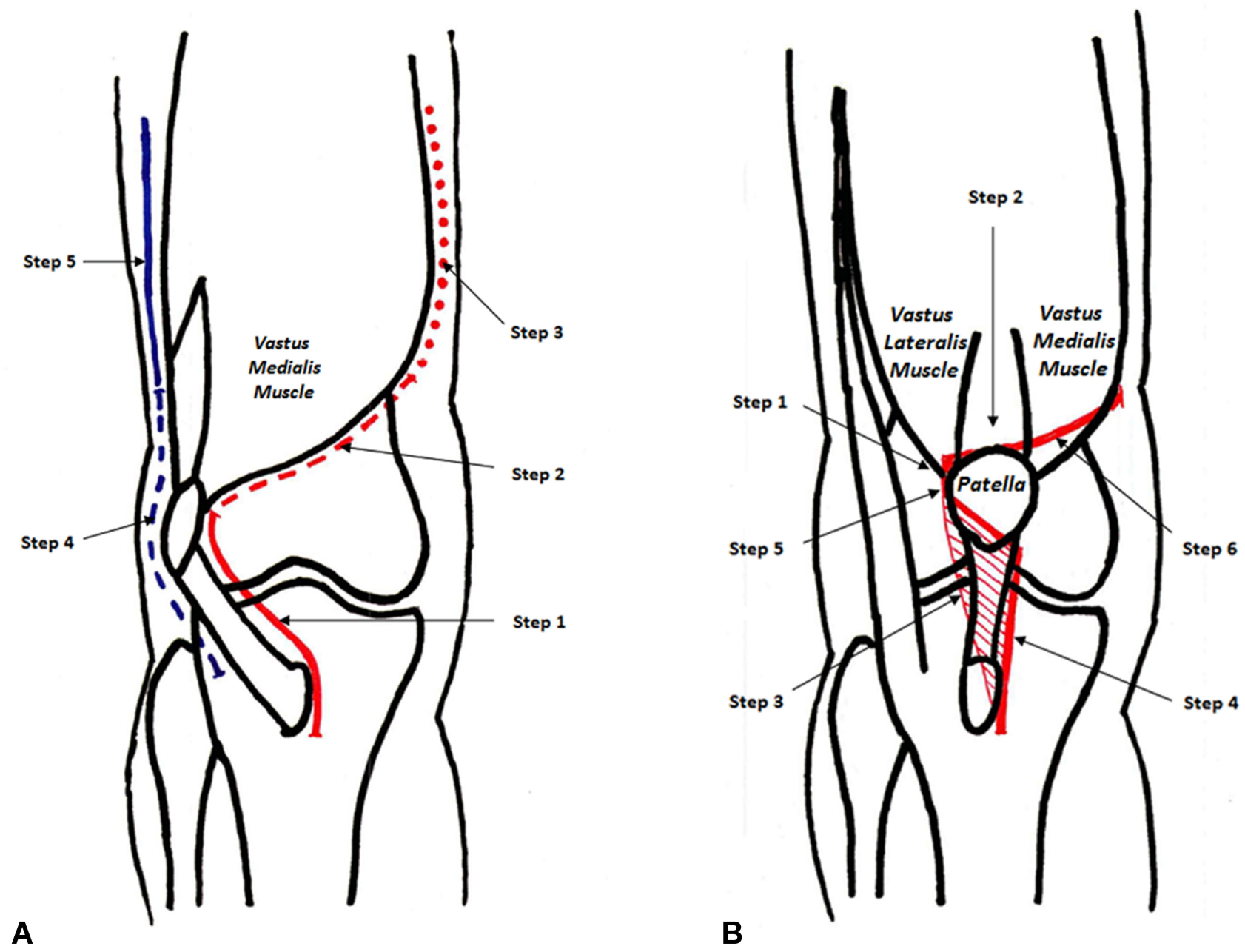

Figure 6 Diagrammatic representation of the soft tissue release performed to correct congenital dislocation of the patella. (A) Step I - Vertical limb of the medial incision (solid red line) made along the medial edge of the patellar tendon to the tibial tubercle; Step 2 - Horizontal limb of the medial incision (dashed red line) taken along the inferior margin of the vastus medialis; Step 3 - Blunt dissection of the vastus medialis from the medial intermuscular septum (dotted red line) approximately $10 \mathrm{~cm}$ proximal to the adductor tubercle; Step 4 - Lateral release of the patellar retinaculum (dashed blue line) performed from inside the knee joint staying lateral to the vastus lateralis; Step 5 - Release of the vastus lateralis (solid blue line) from the lateral intermuscular septum and anterior femur by submuscular dissection. Schematic diagram of the extensor mechanism realignment. (B) Step I - Temporary fixation of the medial retinaculum flap brought laterally and fixed to the superolateral aspect of the patella; Step 2 - Evaluation of the patellar tracking; Step 3 - Resection of the redundant pouch of the medial retinaculum flap pulled over the patellar tendon; Step 4 - Distal medial repair with the suture of the medial capsule and the patellar ligament in a secure manner; Step 5 - Suture of the proximal part of the medial retinaculum flap (pulled over the anterior aspect of the patella) to the extensor apparatus; Step 6 - The medial capsular suture completed proximally. 
informed consent for the case details and images to be published.

\section{Discussion}

Although total knee arthroplasty has become a highly efficient surgical technique for primary osteoarthritis, this procedure remains challenging for the orthopaedic surgeon in patients with spondyloepiphyseal dysplasia mostly due to the unique anatomic considerations. ${ }^{7}$ To the best of our knowledge, only six knee replacements have been reported in patients with spondyloepiphyseal dysplasia. However, de Waal Malefijt et al, Guenther et al, and Raggio et al included these cases in larger series evaluating multiple forms or different joint replacement locations in specific types of skeletal dysplasia. ${ }^{4,7,10}$ Moreover, the published reports focused on the identification of surgery and operative complication risk factors in these individuals; further, the surgical technique details were not elaborated upon. The characteristics of the previously reported cases are summarized in Table 1.

There are two major variants of spondyloepiphyseal dysplasia. ${ }^{11}$ Spondyloepiphyseal dysplasia congenita is inherited in an autosomal dominant manner, although most cases arise due to de novo mutations with no previous family history. Identified at birth by a disproportionately shortened trunk, the afflicted newborns present wide-set eyes, short neck, barrel-shaped chest with pectus carinatum deformity, protuberant abdomen caused by lumbar lordosis, which may be accentuated by hip flexion contracture. Gait problems are common and often attributed to lower extremity deformities, particularly coxa vara and genu valgum. Clubfoot deformity can be seen in patients with congenital variant of the disorder. ${ }^{12}$ Odontoid hypoplasia with atlantoaxial instability may be present with subsequent development of cervical myelopathy at 5-10 years of age. ${ }^{13}$ Respiratory insufficiency has been described in babies with spondyloepiphyseal dysplasia congenita secondary to thoracic dysplasia and secondary to cervical cord compression. ${ }^{14}$ Progressive thoracic kyphoscoliosis develops in late childhood. Cleft palate is also a common clinical feature of the disorder. Ocular anomalies include myopia, retinal detachment, and cataracts. Progressive sensorineural hearing loss may develop in children with spondyepiphyseal dysplasia congenita. ${ }^{12}$ A rare form of congenital variant is associated with nephrotic syndrome. $^{15}$

The second variant, spondyloepiphyseal dysplasia tarda, was described as an X-linked recessive form; however, autosomal recessive and autosomal dominant variants have also been reported. ${ }^{11}$ The individuals afflicted
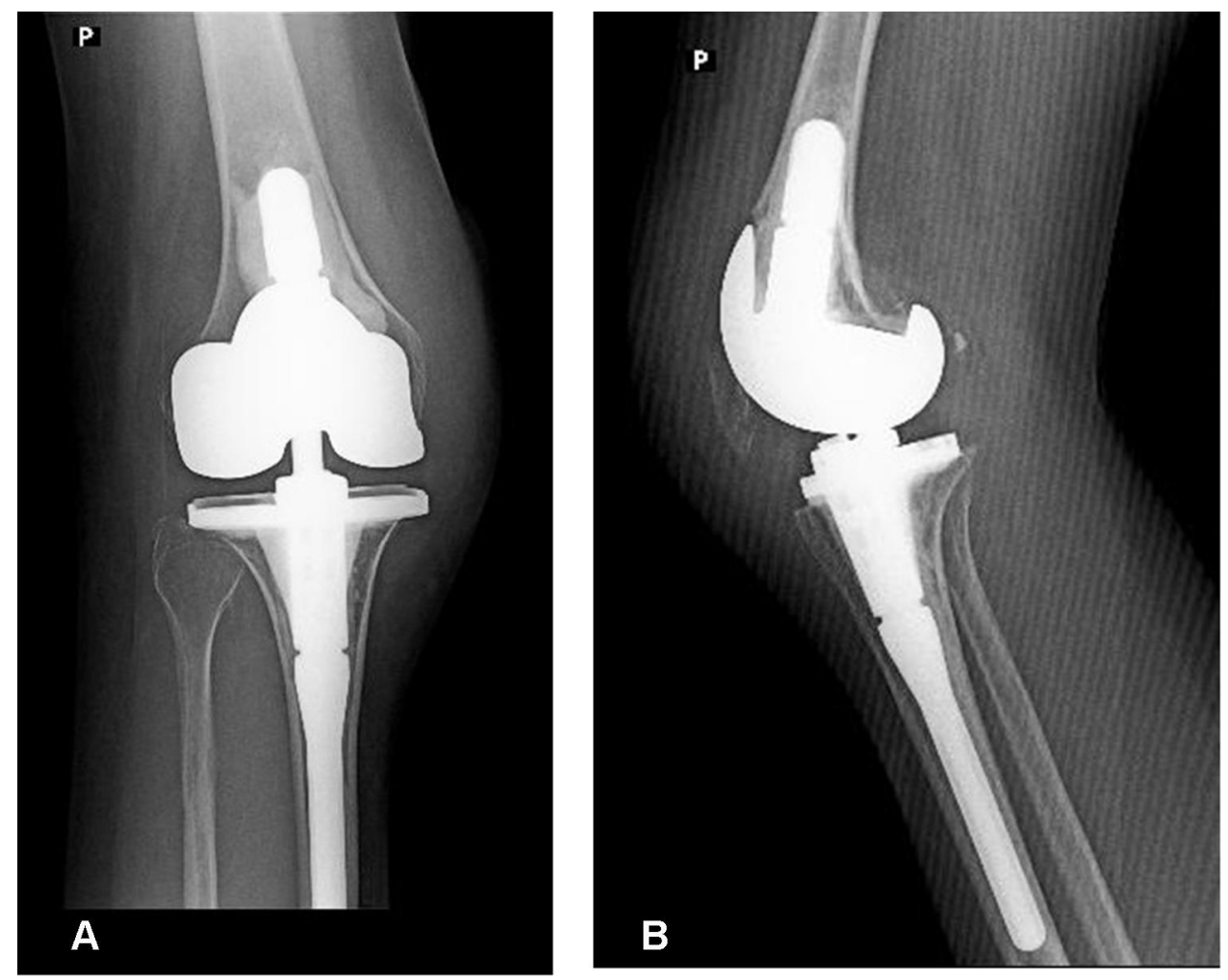

Figure 7 Postoperative radiograph of the right knee: $(\mathbf{A})$ anteroposterior and $(\mathbf{B})$ lateral. 


\begin{tabular}{|c|c|c|c|}
\hline 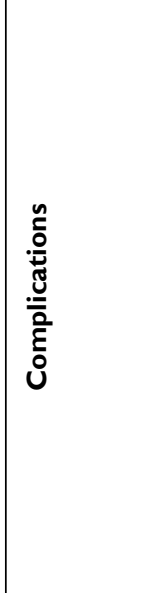 & 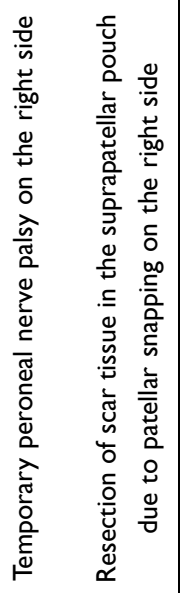 & $\begin{array}{l}\frac{5}{3} \\
0 \\
\frac{5}{5} \\
5\end{array}$ & 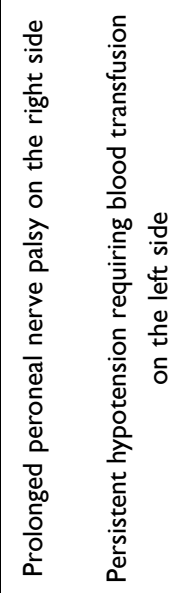 \\
\hline 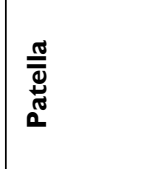 & 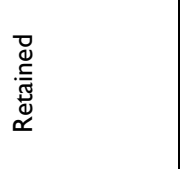 & 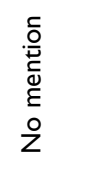 & 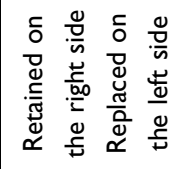 \\
\hline 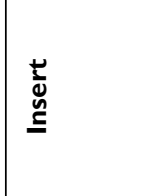 & 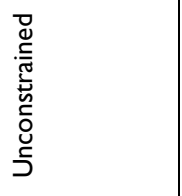 & $\begin{array}{l}\frac{5}{3} \\
0 \\
\frac{5}{5} \\
5\end{array}$ & 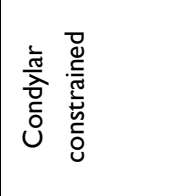 \\
\hline 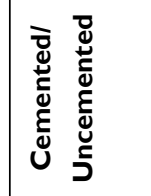 & 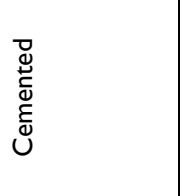 & $\begin{array}{l}\frac{5}{3} \\
0 \\
\frac{5}{5} \\
5\end{array}$ & 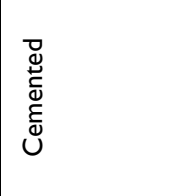 \\
\hline$\stackrel{0}{i n}$ & 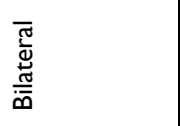 & $\begin{array}{l}\frac{5}{3} \\
\frac{0}{5} \\
\frac{5}{5} \\
5\end{array}$ & 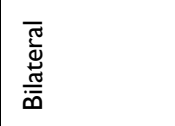 \\
\hline 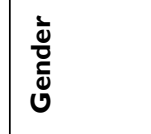 & $\frac{\frac{\omega}{\tilde{N}}}{\frac{\pi}{\Sigma}}$ & $\begin{array}{l}\frac{5}{3} \\
\frac{0}{5} \\
\frac{5}{5}\end{array}$ & 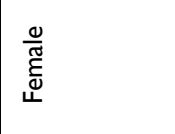 \\
\hline 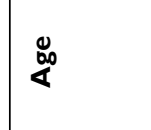 & Hे & $\begin{array}{l}\frac{5}{3} \\
0 \\
\frac{5}{5} \\
\text { 5 }\end{array}$ & $\mathfrak{F}$ \\
\hline$\dot{z} \leftarrow \stackrel{\Phi}{\mathbf{z}}$ & $N$ & $N$ & $N$ \\
\hline $\begin{array}{ll}i \\
\dot{0} \\
\dot{\partial} \\
\dot{z}\end{array}$ & - & $\begin{array}{l}\frac{5}{3} \\
0 \\
\frac{5}{5} \\
5\end{array}$ & - \\
\hline$\stackrel{ \pm}{2}$ & 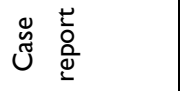 & 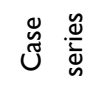 & 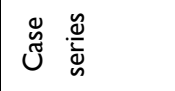 \\
\hline 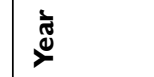 & ঃ্ণ & $\frac{n}{\grave{N}}$ & ర్రి \\
\hline 高 & 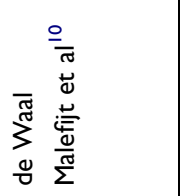 & 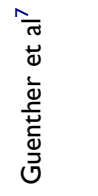 & 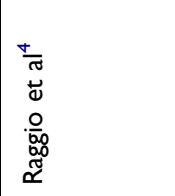 \\
\hline
\end{tabular}

by a milder spondyloepiphyseal dysplasia tarda have a normal appearance at birth presenting a clinical symptom onset between 4 and 10 years of age. Mild disproportionate trunk shortening, barrel-shaped chest, atlantoaxial instability secondary to odontoid hypoplasia, kyphoscoliosis, lumbar lordosis, and epiphyseal involvement in the shoulders, hip, and knees are the common clinical symptoms. Most patients complain about progressive joint pain caused by osteoarthritis, which commonly involves hip, knee and shoulder joints. ${ }^{11}$

A 3- to 6-month time window was recommended for patient- and diagnosis-specific preoperative evaluation to minimize the probability and severity of perioperative and postoperative complications in the patients with skeletal dysplasia. ${ }^{4}$ Further, surgery occurred in tertiary centers able to resolve potential perioperative issues. Anesthesia, radiology, cardiology, pulmonology, neurology, nutrition, psychosocial, and physical therapy consultations are necessary to create an optimal preoperative condition and anticipate postoperative needs. ${ }^{16}$ Typically, the patients with spondyloepiphyseal dysplasia are at risk for atlantoaxial instability. ${ }^{11,13}$ Therefore, preoperative evaluations should include cervical spine imaging. ${ }^{16}$ Anesthesia consultation should be focused on intraoperative airway maintenance, intubation techniques such as fibreoptic intubation, and positioning on the table. ${ }^{4,17}$ Most of the patients with skeletal dysplasia are unable to receive neuraxial anesthesia. ${ }^{18}$

A full-length, weight-bearing lower extremity radiograph is required to properly determine the mechanical and anatomical axis alterations. Bone deformities, small bone caliber, axis deviations, ligament laxity, and soft tissue contractures may require bone resections and prosthetic placement, which differ from knee replacement procedures in patients with primary osteoarthritis. Therefore, the surgeon should thoroughly analyze the patient's CT scans for planning cuts and sizing implants. If preoperative planning reveals particularly small bone anatomy, implants with custom components should be considered. ${ }^{8}$ Moreover, decreased bone density is a frequently omitted problem in patients with skeletal dysplasia. ${ }^{4}$ Poor bone quality increases the risk of iatrogenic intraoperative fracture. In these cases, DEXA scans should be obtained preoperatively and the surgical team should be vigilant for such event during the procedure.

Congenital dislocation of the patella is a rare condition that may be associated with spondyloepiphyseal dysplasia. ${ }^{19}$ There are several reports on knee replacement in cases of 
osteoarthritis with prolonged patellar dislocation. Realignment procedures such as the Insall technique, tibial tubercle osteotomy, and the external rotation setting of the tibia implant in addition to the lateral release of the patellar retinaculum have been previously published. ${ }^{20,21}$ However, in our case, we were unable to obtain a good patella tracking through the lateral release of the patellar retinaculum alone. Moreover, poor bone quality and small bone anatomy precluded tibial tubercle osteotomy. ${ }^{22}$ Based on the preoperative surgical planning, quadricepsplasty was necessary to mobilize and slide the quadriceps muscle during total knee arthroplasty in a patient with spondyloepiphyseal dysplasia and congenital dislocation of the patella.

Spondyloepiphyseal dysplasia commonly requires the careful management of osteoarthritis during a young age. There are few published cases of knee replacement in patients with this diagnosis. The current case report demonstrates the numerous challenges and technical considerations of total knee arthroplasty in patients with spondyloepiphyseal dysplasia. Moreover, special care should be taken to protect the patient from potential complications, and strong emphasis should be set upon careful planning in the preoperative period and vigilant monitoring during and after the surgery.

\section{Ethics Statement}

Written informed consent was obtained from the patient. The approval of the institutional review board was not required in this regard. The patient authorized for both her images and information to be used to this work and allowed them to be published in this journal.

\section{Acknowledgments}

This work was made possible thanks to the grant PROGRES Q40/04 and MHCZ - DRO (UHHK, 00179906). Thanks to Dr. Daniel Diaz for his assistance in proofreading this manuscript.

\section{Disclosure}

The authors report grants from Charles University in Prague. The authors report no other conflicts of interest regarding this work.

\section{References}

1. Mortier GR, Cohn DH, Cornier-Daire V, et al. Nosology and classification of genetic skeletal disorders: 2019 revision. Am J Med Genet A. 2019;179:2393-2419. doi:10.1002/ajmg.a.61366
2. Krakow D, Rimoin DL. The skeletal dysplasias. Genet Med. 2010;12:327-341. doi:10.1097/GIM.0b013e3181daae9b

3. Wyles CC, Panos JA, Houdek MT, Trousdale RT, Berry DJ, Taunton MJ. Total hip arthroplasty reduces pain and improves function in patients with spondyloepiphyseal dysplasia: a long-term outcome study of 50 cases. J Arthroplasty. 2019;34:517-521. doi:10.1016/j.arth.2018.10.028

4. Raggio CL, Yonko EA, Khan SI, et al. Joint replacement in individuals with skeletal dysplasias: one institution's experience and response to operative complications. $J$ Arthroplasty. 2020;35:1993-2001. doi:10.1016/j.arth.2020.04.007

5. Bayhan IA, Abousamra O, Rogers KJ, Bober MB, Miller F, Mackenzie WG. Valgus hip osteotomy in children with spondyloepiphyseal dysplasia congenita: midterm results. J Pediatr Orthop. 2019;39:282-288. doi:10.1097/BPO.0000000000000945

6. Sewell MD, Hanna SA, Muirhead-Allwood SK, Cannon SR, Briggs TWR. Custom cementless THA in patients with skeletal dysplasia results in lower apparent revision rates than other types of femoral fixation. Clin Orthop Relat Res. 2011;469:1406-1412. doi:10.1007/s11999-010-1656-2

7. Guenther D, Kendoff D, Omar M, et al. Total knee arthroplasty in patients with skeletal dysplasia. Arch Orthop Trauma Surg. 2015;135:1163-1167. doi:10.1007/s00402-015-2234-6

8. Kim RH, Scuderi GR, Dennis DA, Nakano SW. Technical challenges of total knee arthroplasty in skeletal dysplasia. Clin Orthop Relat Res. 2011;469:69-75. doi:10.1007/s11999-010-1516-0

9. Chiavetta JB, Parvizi J, Shaughnessy WJ, Cabanela ME. Total hip arthroplasty in patients with dwarfism. J Bone Joint Surg Am. 2004;86:298-304. doi:10.2106/00004623-200402000-00012

10. de Waal Malefijt MC, van Kampen A, van Gemund JJ. Total knee arthroplasty in patients with inherited dwarfism - a report of five knee replacements in two patients with Morquio's disease type A and one with spondylo-epiphyseal dysplasia. Arch Orthop Trauma Surg. 2000;120:179-182. doi:10.1007/s004020050039

11. Chen H. Spondyloepiphyseal dysplasia. In: Chen H, editor. Atlas of Genetic Diagnosis and Counseling. New York: Springer; 2012:2687-2698.

12. Terhal PA, Nievelstein RJ, Verver EJ, et al. A study of the clinical and radiological features in a cohort of 93 patients with a COL2A1 mutation causing spondyloepiphyseal dysplasia congenita or a related phenotype. Am J Med Genet A. 2015;167A:461-475. doi:10.1002/ ajmg.a.36922

13. Al Kaissi A, Ryabykh S, Pavlova OM, et al. The management of cervical spine abnormalities in children with spondyloepiphyseal dysplasia congenita: observational study. Medicine. 2019;98:e13780. doi:10.1097/MD.0000000000013780

14. Harding CO, Green CG, Perloff WH, Pauli RM. Respiratory complications in children with spondyloepiphyseal dysplasia congenita. Pediatr Pulmonol. 1990;9:49-54. doi:10.1002/ ppul.1950090112

15. Rottenberg GT, Shaw DG, Hall CM. Spondyloepiphyseal dysplasia with nephrotic syndrome (Schimke immunoosseous dysplasia). $J$ Pediatr Orthop B. 1997;6:7-10. doi:10.1097/01202412199701000-00002

16. White KK, Bompadre V, Goldberg MJ, et al. Best practices in perioperative management of patient with skeletal dysplasias. $\mathrm{Am}$ J Med Genet A. 2017;173:2584-2595. doi:10.1002/ajmg.a.38357

17. Farag E. Airway management for cervical spine surgery. Best Pract Res Clin Anaesthesiol. 2016;30:13-25. doi:10.1016/j. bpa.2016.01.001

18. Sprung J, Bourke DL, Grass J, et al. Predicting the difficult neuraxial block: a prospective study. Anesth Analg. 1999;89:384-389. doi:10.1097/00000539-199908000-00025

19. Memminger M. Dysplasia spondyloepiphysaria and patella dislocation: a case followed over 10 years. Acta Biomed. 2019;90:326-330. doi:10.23750/abm.v90i3.7247 
20. Dao Q, Chen DB, Scott RD. Proximal patellar quadricepsplasty realignment during total knee arthroplasty for irreducible congenital dislocation of patella. J Bone Joint Sug Am. 2010;92:2457-2461. doi:10.2106/JBJS.H.00812

21. Kumagai K, Kubo M, Maeda T, et al. Total knee arthroplasty for treatment of osteoarthritis with prolonged patellar dislocation. Arthroplasty Today. 2017;3:25-28. doi:10.1016/j.artd.2016.10.001
22. Sponer P, Kucera T. Total knee arthroplasty associated with tibial tubercle and simultaneous femoral and tibial osteotomies for severe extra-articular deformity: a case report. Ther Clin Risk Manag. 2019;15:597-603. doi:10.2147/TCRM.S193888

\section{Publish your work in this journal}

Therapeutics and Clinical Risk Management is an international, peerreviewed journal of clinical therapeutics and risk management, focusing on concise rapid reporting of clinical studies in all therapeutic areas, outcomes, safety, and programs for the effective, safe, and sustained use of medicines. This journal is indexed on PubMed Central, CAS,
EMBase, Scopus and the Elsevier Bibliographic databases. The manuscript management system is completely online and includes a very quick and fair peer-review system, which is all easy to use. Visit http://www.dovepress.com/testimonials.php to read real quotes from published authors. 